\title{
IP DIFFERENTIATED SERVICES OVER A WDM PASSIVE OPTICAL STAR
}

\author{
Josué Kuri \\ Ecole Nationale Supérieure des Télécommunications \\ 46, rue Barrault. 75634 Paris CEDEX 13, France \\ Tel: (+33) 1.45.81.75.70 Fax: (+33) 1.45.81.31.19 \\ kuri@enst.fr \\ Maurice Gagnaire \\ Ecole Nationale Supérieure des Télécommunications \\ 46, rue Barrault. 75634 Paris CEDEX 13, France \\ gagnaire@enst.fr
}

\begin{abstract}
Important recent advances in the field of opto-electronic devices make WDM optical networks a promising solution for the next generation Internet. Such technologies can be considered in the long-haul, metro and access sections of carrier's infrastructures. The main issue in this paper is the design of a Metropolitan Area Network based on a WDM passive optical star well suited for IP differentiated services. Our proposal focuses on both the MAC protocol and buffer management at the end nodes. Various simulation results outline the capacity of our proposal to satisfy the IP Diffserv [1] QoS requirements, as they are specified by the IETF. For that purpose, both Expedited Forwarding [3] and Assured Forwarding [4] are investigated.
\end{abstract}

Keywords: Diffserv, MAN, MAC protocol, WDM, Passive Optical Star.

\section{Introduction}

The passive optical star (POS) is a popular topology for single-hop broadcast-and-select LANs and MANs because of its superiority over other topologies (e.g., bus) in distributing the transmitted power equally to all nodes without inducing much excess loss [6]. Several Media Access Control (MAC) protocols have been proposed to coordinate data

The original version of this chapter was revised: The copyright line was incorrect. This has been corrected. The Erratum to this chapter is available at DOI: 10.1007/978-0-387-35491-0_28 
transmission over parallel WDM channels on this network. The main issue in this paper is the design of an efficient MAC protocol for a POS with support of differentiated services. The protocol incorporates service disciplines and buffer management mechanisms to support specific Diffserv's functional elements called Per-Hop-Behaviors (explained latter in this section).

The Differentiated Services architecture (Diffserv or DS) aims to provide scalable service differentiation in the Internet. A service [1] is defined as set of significant characteristics of packet transmission in one direction across a set of one or more paths within a network. These characteristics may be specified in quantitative or statistical terms of throughput, delay, jitter and/or loss, or may otherwise be specified in terms of some relative priority of access to network resources.

The Diffserv architecture defines a topological and functional distinction between nodes. Boundary nodes, which connect the DS-capable network to another network (either DS or not DS-capable), perform packet classification and traffic conditioning functions (e.g., metering, marking, shaping, policing). Traffic entering the network is classified and possibly conditioned by these nodes; it is then assigned to different relatively coarse collections of packets called behavior aggregates (hereafter, the term aggregate is used to refer to behavior aggregates). Within the DS network, aggregates are serviced by interior nodes in such a way that a specific, externally observable, forwarding behavior is guaranteed to each aggregate at each hop. This forwarding behavior is called PerHop-Behavior (PHB) (actually, both boundary and interior nodes must be able to apply the appropriate PHB to aggregates) and may be specified in terms of the relative access priority to the node's resources (e.g., buffer space, bandwidth) with respect to other PHBs, or in terms of its relative observable traffic characteristics (e.g., allocated link bandwidth, delay, loss) [1]. PHBs are usually specified as groups (PHB group) that share a common constraint applying to each PHB within the group. A single PHB defined in isolation is a special case of a PHB group.

Services meaningful to network users may defined in the Diffserv framework by applying classification and conditioning rules at the network boundary to create aggregates, and coupling these rules with specific PHBs. The Diffserv working group of the IETF currently works on the definition of the Per-Domain-Behavior concept, which is the expected treatment that an identifiable or target group of packets will receive from "edge-to-edge" of a DS domain. A particular PHB (or, if applicable, a list of PHBs) and traffic conditioning requirements are associated with each PDB. Measurable, quantifiable attributes such as jitter and delay are associated with each PDB and these can be used to 
describe what will happen to packets of that PDB as they cross the DS network [2].

Currently, a formal specification is available for two PHB (groups): the Expedited Forwarding (EF) PHB and the Assured Forwarding (AF) PHB group.

The EF PHB [3] is defined as a forwarding treatment for a particular aggregate, say the EF aggregate. The aggregate's departure rate in an EF-capable node must be equal to or greater than a configured rate for the aggregate. The EF aggregate should receive this rate independent of the intensity of any other traffic attempting to pass across the node. Furthermore, it should average at least the configured rate when measured over any time interval equal to or longer than the transmission time of a maximal-sized packet at the configured rate, whereby the maximal packet size is given by the MTU (Maximum Transmission Unit) of the output link.

The AF PHB group [4] (hereafter, referred to as the AF PHB) is a mean to offer different levels of forwarding assurance for IP packets. Four AF classes are defined, where each class is allocated a certain amount of forwarding resources (buffer space and link bandwidth) in the interior nodes. Within each AF class, packets are marked with one of three possible drop precedence levels. In case of congestion, the drop precedence of a packet determines its relative importance within the class. Thus, the forwarding assurance of a packet belonging to an AF class depends on 1) the amount of forwarding resources allocated to the class, 2) the current load of the class and 3) in case of congestion within the class, the drop precedence of the packet. An AF-compliant node should implement four AF classes. Packets in one AF class must be forwarded independently from packets in another AF class. The node must allocate a configurable, minimum amount of forwarding resources (buffer space and bandwidth) to each implemented AF class. Each class should be serviced in a manner to achieve the configured service rate over both small and large time scales.

It should be noted that the EF and AF PHBs are not a mandatory part of the Diffserv architecture, i.e., a node is not required to implement these PHBs in order to be considered Diffserv-compliant.

The rest of the paper is organized as follows: a description of the network architecture is presented in Section 1. The MAC protocol is described in Section 2. Section 3 shows numerical results about QoS parameters obtained through simulations. Finally, Section 4 presents our conclusions and future work. 


\section{NETWORK CONFIGURATION}

We consider a WDM network with $N$ nodes connected through a central passive star coupler (Figure 1). The system has a total of $N+1$ channels, $N$ channels for data transmission and one for control information. The $\lambda_{i}(1 \leq i \leq N)$ data channels and the $\lambda_{c}$ control channel are slot synchronized (Figure 2).

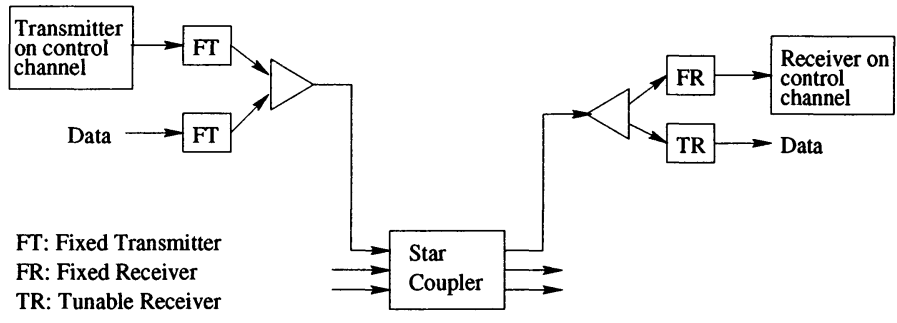

Figure 1 Passive optical star network architecture.

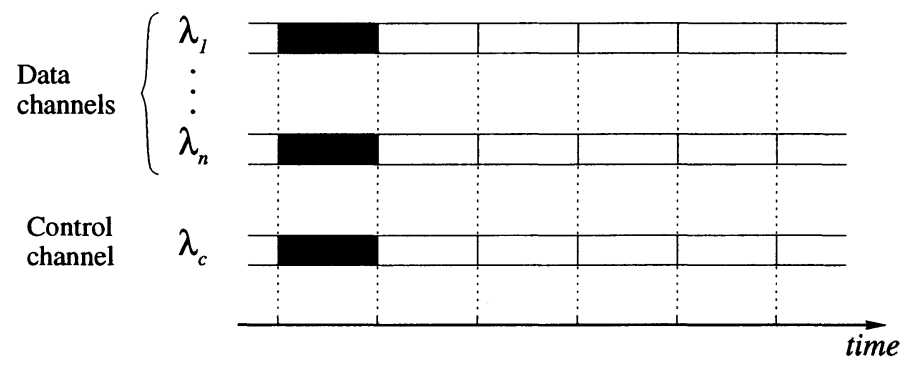

Figure 2 Synchronized slotted structure of the passive optical star.

Each node $N_{i}$ is equipped with a transmitter fixed at a unique wavelength $\lambda_{i}$ for data transmission. A tunable receiver with negligible tuning latency (i.e., the tuning time is significantly smaller than the slot time) and capable of covering all $\lambda_{i}$ channels is used to receive data packets. In addition, each node is equipped with a transceiver fixed at the control channel $\lambda_{c}$. We denote the maximum propagation delay (time) permitted by the system between any pair of nodes by $d_{p}$. Furthermore, we assume that distance ranging is performed by all the nodes.

One of the weak points of the POS topology is the risk of failure of passive coupler at the center of the star. An original topology based on multiple interconnected POSs proposed in [5] prevents this drawback by providing protection and restoration techniques for POSs. 


\section{MAC PROTOCOL}

Several collision-free MAC protocols have been proposed for POS networks (e.g., $[7,8,9])$. These protocols are interesting in metropolitan sized networks, where non-negligible propagation delays make collision handling expensive. Reservation-based collision-free protocols (e.g., $[10,11])$ assign wavelengths to the logical channels between transmitters and receivers on a slot-by-slot basis. Key issues such as the load of the signaling channel, the efficiency of the scheduling algorithm and the impact of propagation delay have been widely investigated for these protocols. In this paper, additional aspects including buffer management mechanisms and service disciplines are considered in order to support service differentiation for IP traffic.

\subsection{DATA UNIT FORMAT}

Slots in the data and control channels are all $L$ bits long. A data unit is the smallest unit of transmission and fits into a slot. A sequence of data units is called a packet. The maximum length of a packet is $M$ data units. Upper layer packets longer than $M$ are split into several MAC-level packets. As depicted in Figure 3, the control channel contains a signaling sub-channel and a reservation sub-channel, which are time-multiplexed on $\lambda_{c}$. Thus, each data unit in the control channel is divided into two fields, SIG and RES, that correspond to the signaling and reservation subchannels, respectively (the utilization of these fields is explained in the following subsections). The SIG field is $S$ bits long and is divided into $N$ mini-slots of length $s=S / N$, one for each node. The RES field is $R=L-S$ bits long and contains a variable number of reservations. A reservation consists of three sub-fields: the destination address, the packet length (in data units) and the Diffserv's PHB ID (PID). The binary representation (base 2) of the values for these fields fits in $\log _{2}(N), \log _{2}(M)$ and $\log _{2}(P)$ bits, respectively. With the total length of a reservation being $r=\log _{2}(N \cdot M \cdot P)$, up to $R / r$ reservations fit into a RES field.

\subsection{SIGNALING}

Reservation-based protocols use a signaling mechanism to convey the transmission requests among the nodes. Scalability is a major concern in such protocols because the bandwidth requirement in the signaling channel is in general a polynomial function of the number of nodes. The SRD (Signaling-Reservation Decoupling) protocol [12] alleviates this problem by distributing information more efficiently in the signaling channel. The 


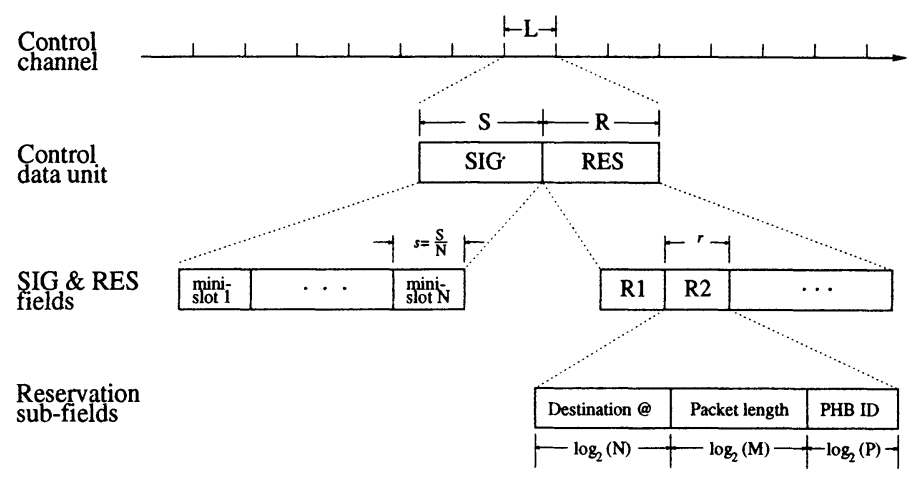

Figure 3 Format of a control data unit

basic idea is to decouple the information indicating that a new packet has to be sent, from the detailed information referring to this packet, namely, the destination address and the length of the packet (in data units). This mechanism allows the signaling channel to be dynamically allocated in such a way that active nodes (those with packets to send) can access the portion of the signaling channel currently unused by inactive nodes. In what follows we present an extended version of the SRD protocol, referred to as $\mathrm{SRD}_{D S}$ (Diffserv-SRD). The protocol consists of the following steps repeated by each node at each time slot (see Figure 4):

1 In time slot $t$, each node $N_{i}$ in the network posts the number of packets, if any, that it received for transmission during $t-1$. The binary representation (base 2) of this number is put into the minislot assigned to $N_{i}$ in the $\mathrm{SIG}_{t}$ field. At the end of $t, \mathrm{SIG}_{t}$ contains the filled mini-slots of all nodes; it propagates then through $\lambda_{c}$.

2 At slot $t+d_{p}$ (the current slot at the time the $\mathrm{SIG}_{t}$ field has completely propagated), the $\mathrm{SIG}_{t}$ field is received and every node is thus aware of how many packets the other nodes whish to transfer.

3 In slot $t+d_{p}+1$, the nodes fill the $\operatorname{RES}_{t+d_{p}+1}$ field with reservations for the packets they announced in the $\mathrm{SIG}_{t}$ field. A Round-Robin service discipline determines their order: each node is allowed to post one reservation in turn. If all nodes have posted their first reservation and if there is still more space available in $\mathrm{RES}_{t+d_{p}+1}$, another round is started, until there is no other reservation to post or no more space left in $\mathrm{RES}_{t+d_{p}+1}$. The signals that did not lead 


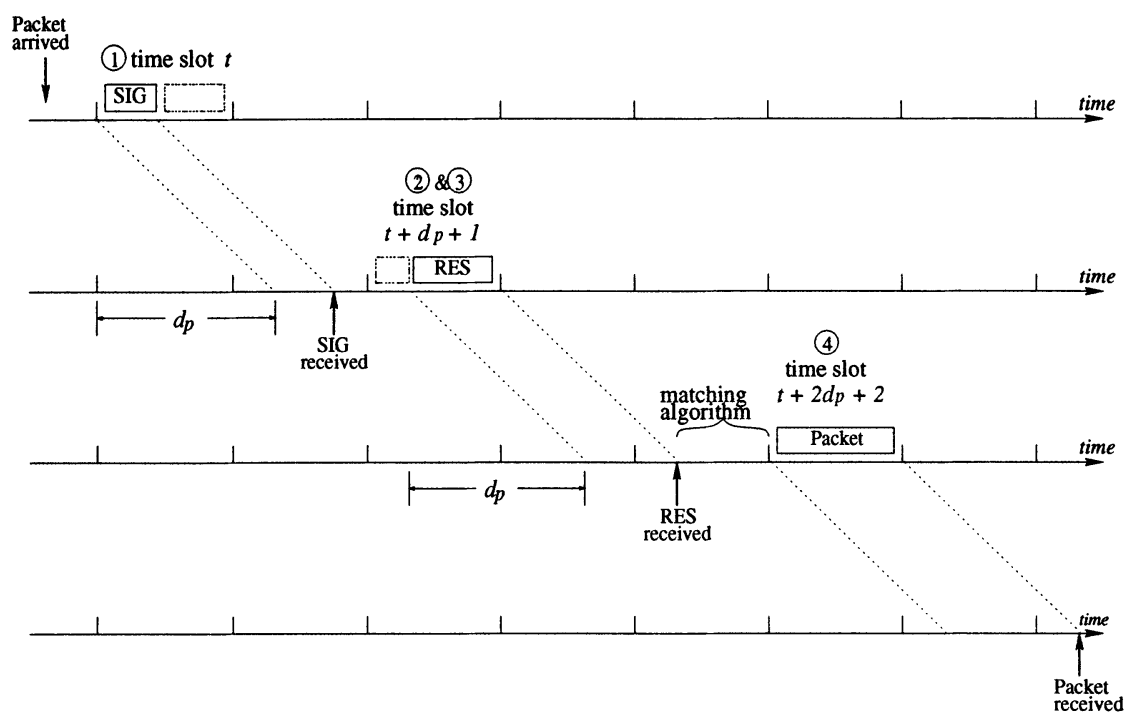

Figure 4 Time diagram of a $\mathrm{SRD}_{D S}$ packet transmission (in this case, the packet fits in a single data unit).

to a reservation are stored at each node in a FCFS queue and considered in the next slot.

4 Once the $\mathrm{RES}_{t+d_{p}+1}$ field propagates to all nodes, at slot $t+2 d_{p}+2$, the nodes become aware of the destinations and lengths of data packets that arrived at their respective transmitting nodes before time slot $t$. This information may be conveniently represented as a backlog matrix, $B(t)=\left(b_{i j}(t)\right)_{i, j=1}^{n}$. $b_{i j}(t)$ represents the set of packets present up to time slot $t$ in node $i$ that are destined to node $j$ and to be sent on wavelength $\lambda_{i}$. Furthermore, each $b_{i j}(t)$ entry is a FCFS queue.

\subsection{ARBITRATION}

At step 4 of the previous algorithm, all the nodes use a common matching algorithm on matrix $B(t)$ to determine the data packets that each of them must transmit, if any, in the following slot. The matching algorithm picks a set of packets among those represented in $B(t)$ such that no two packets in the set have the same source node or destination node. Thus the simultaneous transmission of the set of packets results in no contentions. We use an extended version of the Random Schedul- 
ing Algorithm (RSA) [13] for multiple classes of traffic to compute the matching. The original RSA algorithm was modified so that when the first data unit of a packet longer than a single data unit is scheduled for transmission (i.e., it is matched), all the remaining data units of the packets will be scheduled with no interruption. To support multiple classes of traffic, the algorithm uses five different backlog matrices $B_{c \in 0.4}(t)$, where $B_{0}(t)$ represents the EF traffic and the other the traffic of each AF class, from 1 to 4 . The algorithm is presented in Fig. 5.

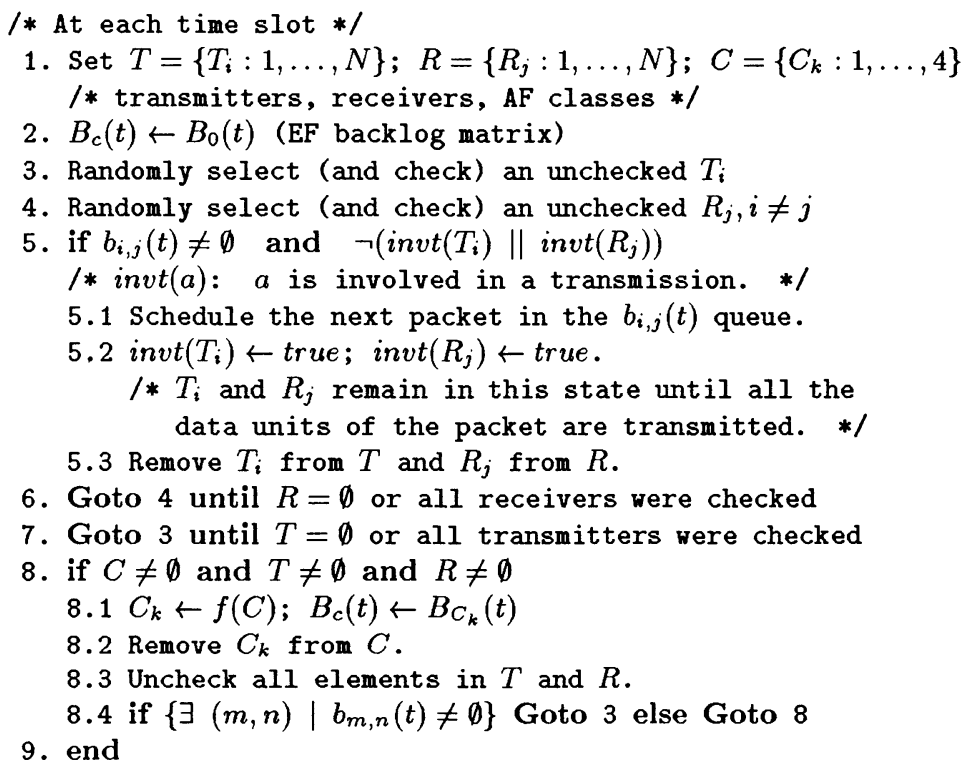

6 . Goto 4 until $R=\emptyset$ or all receivers were checked

7. Goto 3 until $T=\emptyset$ or all transmitters were checked

8. if $C \neq \emptyset$ and $T \neq \emptyset$ and $R \neq \emptyset$

$8.1 C_{k} \leftarrow f(C) ; B_{c}(t) \leftarrow B_{C_{k}}(t)$

8.2 Remove $C_{k}$ from $C$.

8.3 Uncheck all elements in $T$ and $R$.

8.4 if $\left\{\exists(m, n) \mid b_{m, n}(t) \neq \emptyset\right\}$ Goto 3 else Goto 8

9. end

Figure 5 The packet-aware multiple class RSA matching algorithm

Step 8.1 of the algortihm is executed 4 times at most. On each execution, the function $f(C)$ selects a particular class $C_{k}$. The proportion of times (with respect to the total number of times the algorithm is executed) that $C_{k}$ is selected in the first execution of the step is equal to the proportion of resources allocated to the class; the same applying for the second, third and fourth executions of the step.

\subsection{BUFFER MANAGEMENT AND SERVICE DISCIPLINE}

In what follows, we describe the buffer management mechanisms and the service discipline considered in the MAC protocol to support the $\mathrm{EF}$ 
and AF PHBs requirements as defined in RFC 2598 [3] and RFC 2597 [4], respectively.

It should be noted that a PHB is defined as the externally observable forwarding behavior applied at a Diffserv-compliant node to a Diffserv behavior aggregate [1]. The POS for which our MAC protocol is designed for, is a broadcast-and-select, single-hop network. So, we consider the whole POS network as topologically and functionally equivalent to a interior node of a multi-hop network, where the PHBs are implemented. Furthermore, the "externally observable" forwarding behavior is considered in the POS at the egress of the network, that is, at the sink nodes (Figure 6). Because of this fundamental consideration, we assume that the traffic entering the network is already classified into aggregates and conditioned.

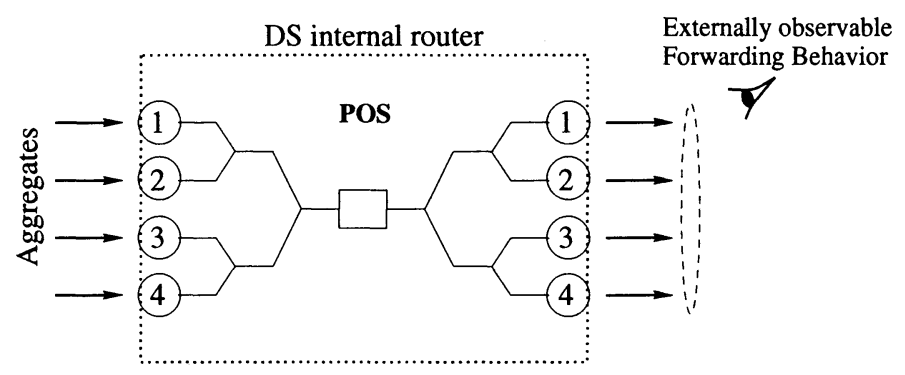

Figure 6 The POS as a DS interior node.

In order to meet the specific PHB requirements, we begin by identifying the resources to be allocated in the POS to the EF and AF aggregates. Recall from the presentation of the signaling protocol that, for a packet to get transfered, it must be first queued at the source node; then signaled in the SIG field. Once the SIG field is broadcast, the packet must access a dynamically allocated reservation in the RES field to convey its detailed information. When the reservation reaches all the nodes, it must be scheduled by the matching algorithm before the packet is finally transmitted (i.e., all the data units that conform the packet). From this recapitulation we identify the required resources: the transmission queue of the source node, a share of the signaling channel and the scheduling of the packet by the matching algorithm.

The transmission queues in a node $N_{i}$ are arranged as depicted in Figure 7. There is one queue dedicated to EF traffic and four queues for AF traffic, one per AF class. Each queue, either EF or AF, is logically divided into three sections by two pointers (see the AF class 4 queue 
at the bottom of the figure). The leftmost section contains the packets waiting to be signaled i.e., the packets waiting to be indicated in the next SIG field (the node's mini-slot in the next SIG field will contain the number 2). The section in the center contains the packets waiting for a reservation in the RES sub-channel. Finally, the rightmost section contains the packets waiting to be scheduled (i.e., waiting for a "match" of the common matching algorithm) before being transmitted. Thus, the right side pointer moves to the right when a packet is signaled (SIG) and the left side pointer moves also to the right when the reservation (RES) of a packet has been sent.

The sources feeding the node $N_{i}$ may be grouped into $N-1$ sets according to their destination node $N_{j}$, where $i \neq j$ and $1 \leq j \leq N$. Each $\mathrm{S}_{i \rightarrow j}$ set may contain zero or more EF sources and zero or more sources of each AF class.

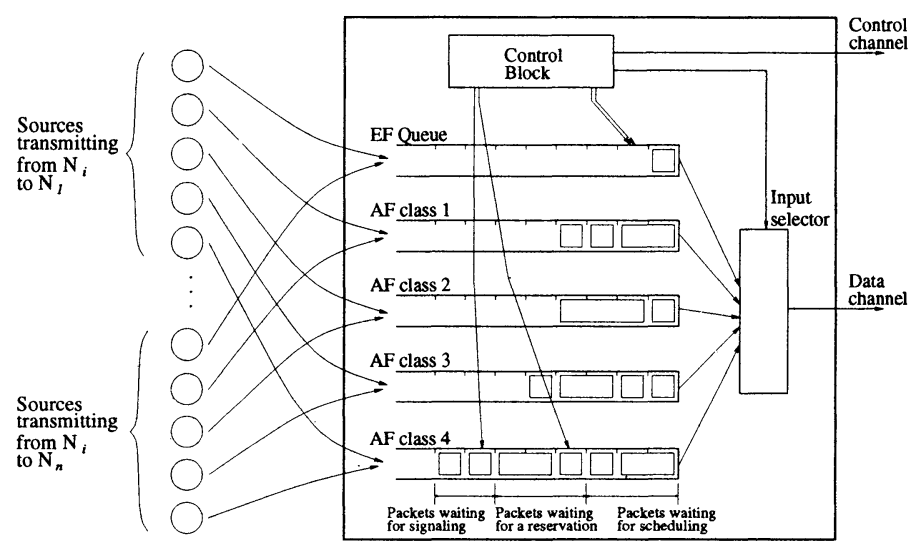

Figure $7 \mathrm{SRD}_{D S}$ node configuration with $\mathrm{EF} / \mathrm{AF}$ support.

The buffer management mechanism must be implemented in the queues in order to avoid potential congestions. Upon a packet arrival, the mechanism determines whether the packet is accepted or not. The EF queue implements tail dropping (dropping in this queue should never occur because the arriving traffic is supposed to be rate-limited and shaped before entering the network. Furthermore, it is served with static priority), whereas AF queues use a Random Early Drop (RED) algorithm [14]. The RED algorithm aims to achieve high throughput and low delay, mainly under responsive traffic (e.g., TCP), by controlling the average queue size. The average size is kept low, while fluctuations in the actual size are allowed to accommodate bursty traffic and transient 
congestions. The RED algorithm calculates the average queue size $q_{a}$ at each packet arrival using a low-pass filter with an exponential weighted moving average. The $q_{a}$ value is compared to two queue size thresholds $\min _{t h}$ and $\max _{t h}$. When $q_{a}<m i n_{t h}$, the packet is accepted. When $q_{a} \geq \max _{t h}$, the packet is dropped. If $\min _{t h} \leq q_{a}<\max _{t h}$, the packet is dropped with probability $p_{a}=f\left(q_{a}, P_{\max }\right)$, where $P_{\max }$ is the maximum dropping probability. We extend the basic RED algorithm to accommodate three levels of dropping precedence within the AF queue, namely, low precedence (green), i.e., packets have low probability of being dropped; medium precedence (yellow) and high precedence (red). A tuple $<\min _{t h}, \max _{t h}, P_{\max }>$ is associated with each dropping precedence level (Figure 8) in such a way that the total buffer space allocated to the AF class is a function of the values of the three tuples.

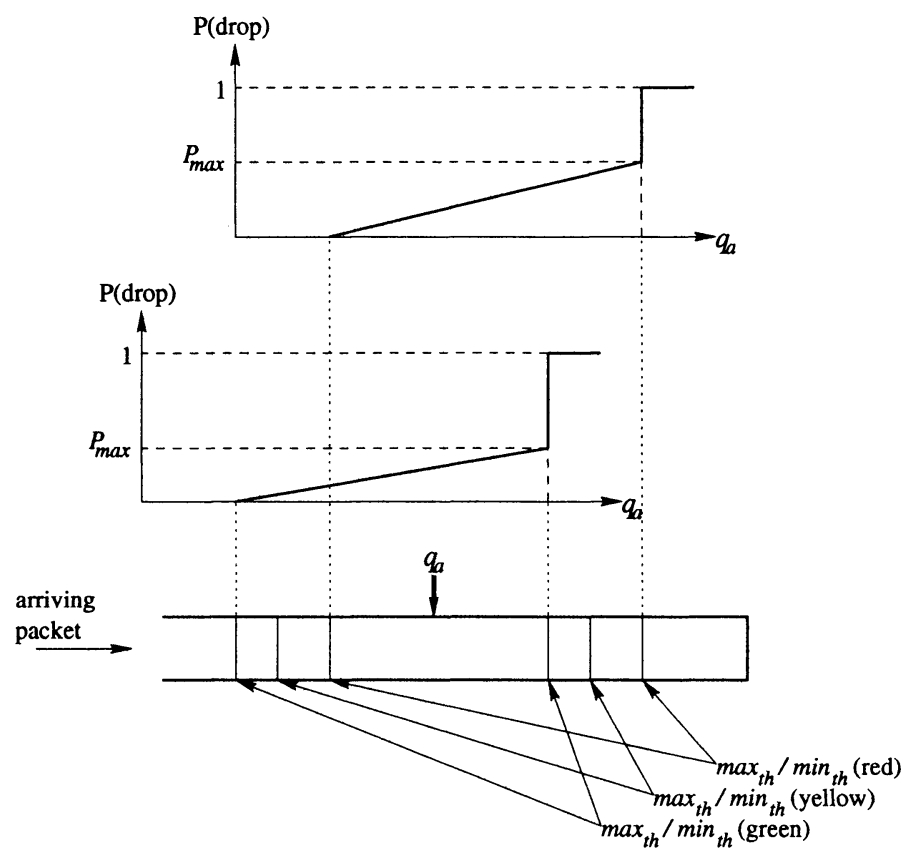

Figure 8 RED queue with three dropping precedence levels.

Once an arriving packet is accepted in the queue, it must be signaled through the control channel. At this point, the node counts the total number of packets that arrived at all its queues in the previous slot and posts this value in its assigned mini-slot of the SIG field. When the SIG field is received, the RES field of the next slot is filled up with reser- 
vations for the signaled packets. If all nodes have packets to transfer, each node is allocated one out of $N$ reservations in the RES field. Each node must in turn distribute its allocated signaling among the packets of its $\mathrm{EF}$ and AF queues. This distribution has to be done so that the bandwidth requirement of each PHB, namely, the EF and the four AF classes, is conveniently signaled. Thus, the service rule is first to post reservations for packets in the EF queue up to exhaustion. The remaining reservations are then distributed among the packets in the AF queues using a Weighted Round Robin service discipline, where the weight of each queue corresponds to the share of bandwidth for the respective AF class. Here, note that the average size of packets in an AF class must be considered when calculating the weight of its queue; otherwise, if two queues have the same weight but different average packet size, the one with the largest average will get actually more bandwidth.

The final step of the MAC protocol, before a packet is actually transmitted, is the scheduling of the intended transmission by the matching algorithm. The Random Scheduling Algorithm must also satisfy the bandwidth requirements of both PHBs. The algorithm first attempts to schedule transmissions of EF packets. The AF transmissions are scheduled, according to the weight of their respective class, only when there are no more possible matches in the EF backlog matrix $B_{0}(t)$, and there are still sources and destinations available (see Figure 5).

\subsection{TRAFFIC CONDITIONING AND MARKING}

The traffic entering the network must be conditioned in order to meet the expected requirements. Regarding the EF traffic, it is clear that, since it is served with static priority, it must be hard-limited to a configured peak rate and shaped when entering a node, so that bursts are not injected in the network and the AF aggregates do not starve. Starvation implies high access delay and high packet loss of $A F$ traffic due to congestion.

For the AF PHB, a certain amount of forwarding resources (buffer space and bandwidth) is allocated to each class. More precisely, the signaling allocation of the node and the transmissions scheduled by the RSA algorithm for AF traffic are proportionally distributed among the four AF classes. Based on the proportion of allocated resources, a nominal rate for each AF aggregate is specified. A rate meter [15] is used to calculate the instantaneous rate of packets arriving at a given $\mathrm{AF}$ queue. If the measured rate is below or slightly above the nominal rate, the arriving packet is marked with the lowest dropping precedence (green). 
If the rate is above the nominal rate but below a first threshold, it is marked with medium precedence (yellow); finally, if the rate is above this threshold, the packed is marked with the highest dropping precedence (red). Once the packet has been marked, the RED algorithm is applied to determine if the packet is either accepted or not in the AF queue, based on the average queue size and the queue size thresholds defined for the color of the packet.

\section{PERFORMANCE EVALUATION}

In this section, we experimentally evaluate the ability of the MAC protocol to meet the requirements of the EF and AF PHBs. In addition, we present results illustrating the impact of the interaction of $\mathrm{EF}$ and AF PHBs on traffic metrics such as packet loss.

\subsection{SIMULATION AND TRAFFIC MODELS}

Table 1 shows the parameters of the network considered in the simulations.

Table 1 Network parameters used in simulations

\begin{tabular}{lr}
\hline Parameter & Value \\
\hline Nodes & 8 \\
Channel rate & $2.5 \mathrm{Gbit} / \mathrm{s}$ \\
Slot size & $384 \mathrm{bits}$ \\
Propagation delay & $10 \mathrm{slots}$ \\
\hline
\end{tabular}

The sources feeding the EF aggregate generate fixed-length packets of 48 bytes at constant bit rate. The sources generating traffic for the AF classes are characterized by an empirical model of LAN traffic introduced in [16]. Packet interarrival time is assumed to be exponential, with a mean rate $\lambda$ and the packet length is distributed as in tables 2 and 3 . For an EF source, its offered load is the ratio of its constant bit rate to the rate of the data channel over which it send its packets. For AF traffic, the offered load of a source is the ratio of its average rate to the rate of the data channel over which it sends its packets.

The packet's length distribution of table 2 represents typical WWW traffic, whereas the distribution in table 3 represents a traffic pattern typical of bulk transfers (e.g., FTP, e-mail, etc.). 
Table 2 WWW traffic's packet length distribution (bytes)

\begin{tabular}{lrrrrrr}
\hline Packet length & 64 & 128 & 256 & 512 & 1024 & 1518 \\
Probability & 0.6 & 0.06 & 0.04 & 0.02 & 0.25 & 0.03 \\
\hline
\end{tabular}

Table 3 FTP traffic's packet length distribution (bytes)

\begin{tabular}{lrrrrrr}
\hline Packet length & 64 & 128 & 256 & 512 & 1024 & 1518 \\
Probability & 0.06 & 0.06 & 0.04 & 0.02 & 0.25 & 0.57 \\
\hline
\end{tabular}

\subsection{EF CONFORMANCE}

We first verify the bounds of the rate that can be guaranteed to EF sources. We set $N=4$ nodes in the network as active, each one with sources transmitting to the other three active nodes; thus, there are $N(N-1)$ possible unidirectional $\mathrm{EF}_{i \rightarrow j}$ transmissions in the network. Sources generate traffic at $2500 \mathrm{Mbit} / \mathrm{s}$ (i.e., the channel rate), which means that all the EF queues are always backlogged. Figure 9 depicts the rate attained by the $\mathrm{EF}$ transmissions over time (measured in sink nodes).

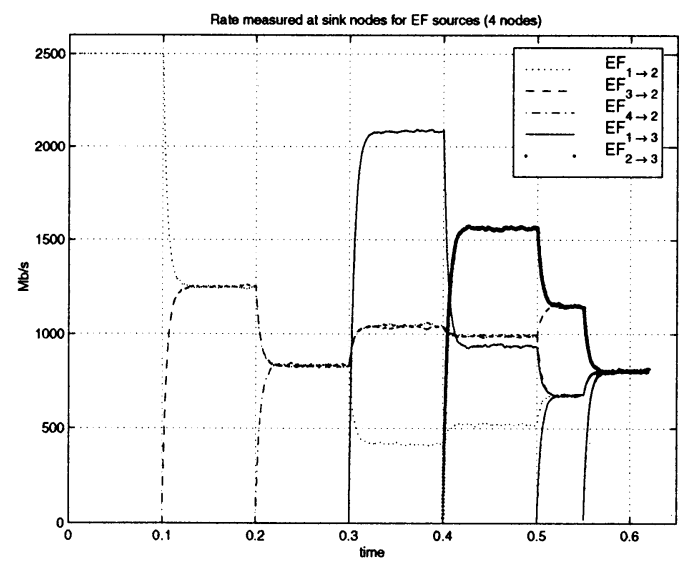

Figure 9 Rate of EF sources measured at sink nodes $(\mathrm{N}=4)$. 
The source feeding the $\mathrm{EF}$ transmission from node 1 to node $2, \mathrm{EF}_{1 \rightarrow 2}$, is turned on at $t=0 \mathrm{~s}$. Since it is the only active transmission, it gets all the resources in the network and attains the maximal rate of $2500 \mathrm{Mbit} / \mathrm{s}$. On $t=0.1 \mathrm{~s}$, the source feeding the $\mathrm{EF}_{3 \rightarrow 2}$ transmission is turned on. At this point, the receiver of node 2 must be tunned back and forth to the $\lambda_{1}$ and $\lambda_{3}$ channels (i.e., the channels transmitters 1 and 3 are fixed to, respectively) so, each transmission attains a half of the maximal rate. On $t=0.2 \mathrm{~s}$, the $\mathrm{EF}_{4 \rightarrow 2}$ transmission becomes active and the receiver of node 2 is "shared" among the three transmissions; each transmissions gets a third of the maximal rate $(\approx 833.33 \mathrm{Mbit} / \mathrm{s})$. On $t=0.3 \mathrm{~s}$, the $\mathrm{EF}_{1 \rightarrow 3}$ transmission is active. Here, note that the rate of both $\mathrm{EF}_{3 \rightarrow 2}$ and $\mathrm{EF}_{4 \rightarrow 2}$ increases, whereas $\mathrm{EF}_{1 \rightarrow 2}$ achieves a lower rate. This is because now the transmitter of node 1 must be "shared" between $\mathrm{EF}_{1 \rightarrow 2}$ and the recently introduced $\mathrm{EF}_{1 \rightarrow 3}$ transmission. Consequently, $\mathrm{EF}_{1 \rightarrow 2}$ gets a lower rate $(\approx 416.66 \mathrm{Mbit} / \mathrm{s})$, whereas $\mathrm{EF}_{3 \rightarrow 2}$ and $\mathrm{EF}_{4 \rightarrow 2}$ take advantage of the released bandwidth at receiver 2 . On the other hand, the introduced transmission $\mathrm{EF}_{1 \rightarrow 3}$ attains a rate of $\approx 2083.3 \mathrm{Mbit} / \mathrm{s}$ which, added to the rate of $\approx 416.66 \mathrm{Mbit} / \mathrm{s}$ of the $\mathrm{EF}_{1 \rightarrow 2}$, yields the maximal transmission rate $(2500 \mathrm{Mbit} / \mathrm{s})$ of the node 1 's transmitter. On $t=0.4 \mathrm{~s}$ and $t=0.5 \mathrm{~s}$, other two transmissions become active. Finally, on $t=0.55 \mathrm{~s}$, the six remaining transmissions are activated. At this point, the minimum guaranteed rate of $C \cdot 1 /(N-1)$ is attained for each possible connection in the network.

According to RFC 2598, EF traffic should receive the configured rate independent of the intensity of any other traffic attempting to transit the node. We are interested on the impact of AF traffic on EF connections. Figure 10 shows the instantaneous rate of EF sources observed at sink nodes. The sources generate data-unit-long packets at a) $200 \mathrm{Mbit} / \mathrm{s}, \mathrm{b}$ ) $300 \mathrm{Mbit} / \mathrm{s}$, and c) $400 \mathrm{Mbit} / \mathrm{s}$ with WWW background load. The global load (EF and $\mathrm{AF}$ ) is fixed to $72 \%$ of the network capacity (the load offered by all the sources over all data channels). In a), the measured rate shows little jitter, whereas the rate in c) is highly variable. In the MAC protocol, the rate of a transmission directly depends on the number of matches per time unit that the RSA algorithm allocates to it. Let us recall that a match of a transmitter-receiver is infeasible if either the transmitter or the receiver is involved in another transmission at the time that the match is attempted. This occurs when:

1 The transmitter/receiver was assigned in a previous time slot to another transmission which has not finished yet.

2 The transmitter/receiver was already assigned to another transmission, either $\mathrm{EF}$ or $\mathrm{AF}$, in the current time slot. 
Note that in the first case, an EF transmission can be blocked only by an $\mathrm{AF}$ transmission since all the $\mathrm{EF}$ packets are one data unit long (i.e., they are transmitted in one slot time). In the second case, an EF transmission can be blocked only by another EF transmission because of the static priority mechanism. The probability of both events proportionally increases with the rate of the EF transmissions.

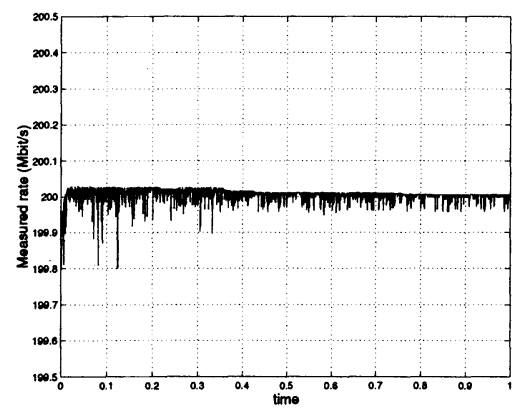

(a)

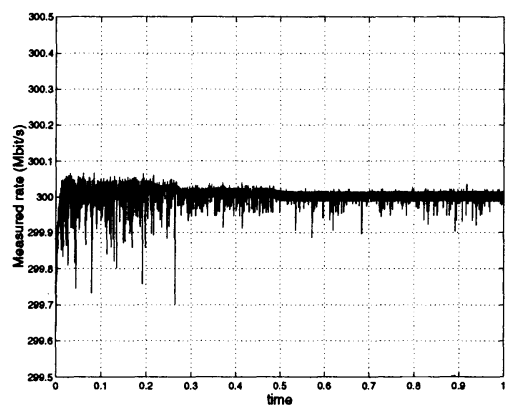

(b)

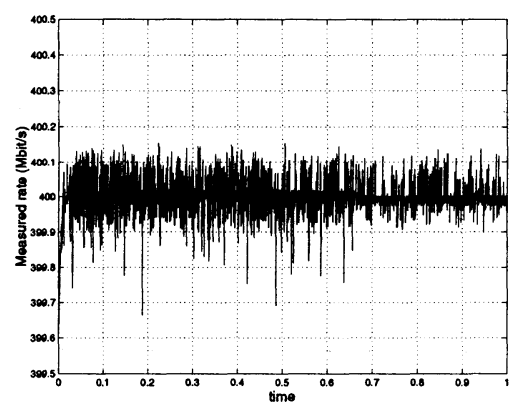

(c)

Figure 10 Rate of EF sources with background traffic measured at sink nodes. a) $\mathrm{EF}=200 \mathrm{Mbit} / \mathrm{s}, \mathrm{b}) \mathrm{EF}=300 \mathrm{Mbit} / \mathrm{s}, \mathrm{c}) \mathrm{EF}=400 \mathrm{Mbit} / \mathrm{s} . \quad \rho=0.72$

\subsection{AF CONFORMANCE}

An AF-compliant node must allocate a configurable, minimum amount of forwarding resources (buffer space and bandwidth) to each implemented AF class. 
We carried out experiments to evaluate the relative priority of access to network resources of the four AF classes. We set four AF source for each possible unidirectional transmission in the network (with $N=8$ nodes, there are 56 transmissions), thus a total of 224 sources feed the network. The offered load is equally divided among the sources. In the nodes, the four AF classes are allocated the same amount of buffer space. To evaluate the proportional allocation of resources (i.e., signaling channel and RSA matches), we set the following proportion to each class:

Table 4 Proportional allocation of forwarding resources among AF classes.

\begin{tabular}{cc}
\hline AF class & weight \\
\hline 1 & 0.4 \\
2 & 0.3 \\
3 & 0.2 \\
4 & 0.1 \\
\hline
\end{tabular}

Clearly, in a scenario where all the AF classes receive the same traffic load, a class with a relatively low proportion of resources will be more susceptible to congestions than classes with more allocated resources, yielding higher values of packet loss probability. The loss probability of a class is associated with its forwarding assurance.

Figure 11 shows the packet loss probabilities of the four AF classes. The left side of the figure shows the probabilities when the AF traffic follows the packet's length distribution of Table 2 (WWW traffic). The right side depicts the probabilities when bulk transfer's packet length's distribution is used. We observe in both cases that a differentiation among classes in terms of forwarding assurance can be attained by the protocol. We observe further that bulk transfers achieve lower drop probabilities than WWW traffic.

\subsection{EF/AF INTERACTION}

Useful behavioral distinctions are mainly observed when multiple behavior aggregates compete for buffer and bandwidth resources in the network. The proposed mechanism for the EF PHB (i.e., priority queue) allows unlimited preemption of other traffic. We evaluate the damage that $\mathrm{EF}$ traffic could inflict on $\mathrm{AF}$ traffic in order to determine the limits that must be imposed on it (e.g., maximum rate). Concretely, preemption of $\mathrm{AF}$ traffic by $\mathrm{EF}$ traffic induces, in the worst case, significant congestion in the AF queues of the node. This prob- 


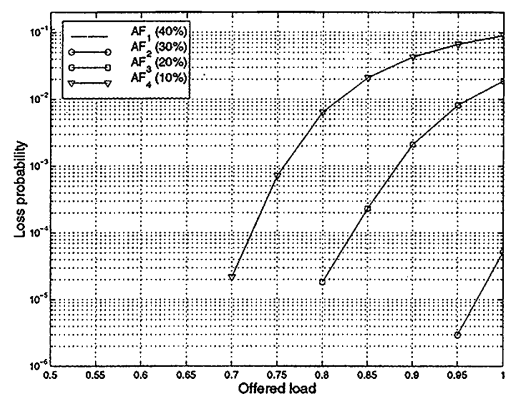

(a)

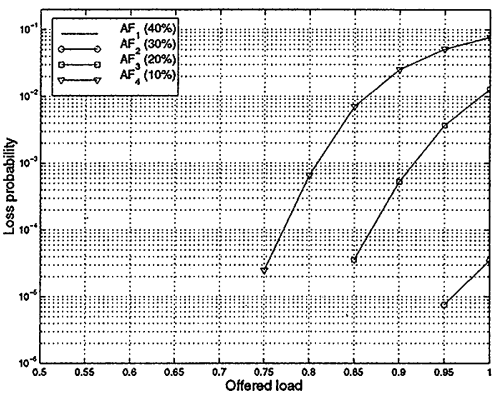

(b)

Figure 11 Packet loss probability of the AF classes with a) WWW traffic and b) FTP traffic.

lem implies an increment of the packet loss probability. For the results in Figure 12, class $\mathrm{AF}_{1}$ is allocated $40 \%$ of forwarding resources (i.e., signaling channel and RSA matches), class $\mathrm{AF}_{2} 30 \%$, class $\mathrm{AF}_{3}$ $20 \%$ and class $\mathrm{AF}_{4}$ the remaining $10 \%$. The RED parameters are the same for the three dropping precedence levels of the four AF queues $<\min _{t h}: 22, \max _{t h}: 32, P_{\max }: 0.5>$. Traffic for each AF class is set to $12.5 \%$ of the offered load, thus, the four AF classes receive altogether $50 \%$ of the offered load. EF traffic is injected in $5 \%$ steps of the total offered load and the packet loss probability is observed for the AF classes at each step.

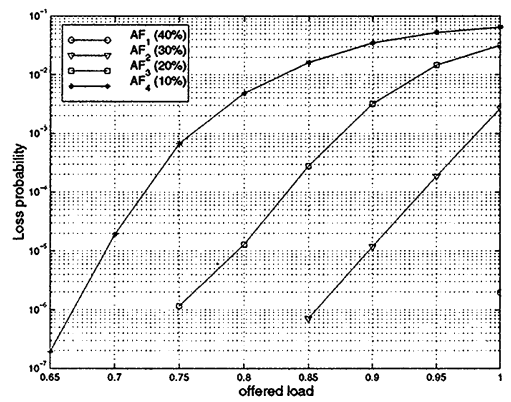

Figure 12 Packet loss probability of the AF classes with background EF traffic. 
By comparing the loss probability values of Figures 11 and 12, we observe that the EF aggregate induces significant congestions in the AF queues and, consequently, packet loss.

\section{CONCLUSIONS AND FUTURE WORK}

The new Internet generation requires high capacity networks able to provide a certain level of QoS guarantees both in the core and metropolitan access networks. In this paper we have presented a MAC protocol for a WDM POS with support for the EF and AF PHBs as defined in the IETF Diffserv architecture. Signaling, arbitration, buffer management and service discipline functions interact to support service differentiation. These various issues have been investigated through simulations.

Regarding the EF PHB, the maximum rate that can be guaranteed to a particular EF transmission in the network depends on the number and rate of other transmissions concurrently using the transmitter and receiver involved in the transmission. Moreover, jitter induced in the $\mathrm{EF}$ transmissions is mainly due to the intensity of the EF traffic itself, rather than to the intensity of other traffic.

For the AF PHB group, the proportional allocation of forwarding resources (i.e., the signaling channel and scheduling of transmissions) among classes has been evaluated. Under the same load conditions (i.e., each $\mathrm{AF}$ class receiving the same amount of traffic) and the same buffer space allocation, packets belonging to classes with more allocated resources experience lower loss probability than packets belonging to the classes with less resources. In this context, two traffic patterns have considered: WWW and bulk data transfers.

The main contribution of this paper is the introduction of service differentiation support in the MAC protocol. A critical aspect that must be further studied is the RSA scheduling algorithm. Its random nature induces distortion in the observed forwarding behaviors. Deterministic scheduling algorithms should be also considered.

Finally, the optimization of the RED's parameters for buffer management in this network must be investigated under more realistic traffic conditions (e.g., responsive/non-responsive traffic). Usually, RED is utilized to simplify the congestion control job required in transport protocols like TCP. Each time a packet is dropped, the concerned TCP session must adjust its rate. TCP flows in an aggregate may be punished for their good behavior in the presence of non-congestion-sensitive flows like UDP within the same aggregate. 
In our coming studies, the service disciplines and buffer management techniques used in this paper will be further exploited for the design of Diffserv edge routers in the context of a cloud of optical switch-routers.

\section{References}

[1] S. Blake, D. Black, M. Carlson, E. Davies, Z. Wang and W. Weiss: An Architecture for Differentiated Services, IETF RFC 2475, December 1998.

[2] K. Nichols and B. Carpenter: Definition of Differentiated Services Per Domain Behaviors and Rules for their Specification, work in progress, (draft-ietf-diffserv-pdb-def-00.txt), June 2000.

[3] V. Jacobson, K. Nichols and K. Poduri: An Expedited Forwarding PHB, IETF RFC 2598, June 1999.

[4] J. Heinanen, F. Baker, W. Weiss and J. Wroclawski: Assured Forwarding PHB Group, IETF RFC 2597, June 1999.

[5] M. Gagnaire and T. Stroesslin: A WDM Network with Flexible Topology for IP and ATM Traffic Transport, 4th IFIP ONDM'2000 Conference, Athens, Greece, February 2000.

[6] R. Ramaswami and K. Sivarajan: Optical Networks: a practical perspective, Morgan Kaufmann Publishers, 1998.

[7] D. Levine and I. Akyildiz: PROTON: A Media Access Control Protocol for Optical Networks with Star Topology, IEEE/ACM Transactions on Networking, vol. 3, no. 2, April 1995, pp. 158-169.

[8] M. Guizani: High-Speed Protocol for an All-Optical Packet Switched Metropolitan Area Network, International Journal of Network Management, vol. 7, no. 1, January/February 1997, pp. 9-17.

[9] P. Humblet, R. Ramaswami and K. Sivarajan: An Efficient Communication Protocol for High-Speed Packet-Switched Multichannel Networks, IEEE Journal on Selected Areas in Communications, vol. 11, no. 4, May 1993, pp. 568-578.

[10] D. Levine and I. Akyildiz: A Reservation and Collision-Free Media Access Protocol for Optical Star Local Area Networks, Proceedings of IEEE GLOBECOM 1994, San Francisco, CA, November/December 1994 , pp. 567-571.

[11] E. Dinan and M Gagnaire: PCSA: A MAC Protocol for Single-hop Wavelength Division Multiplexed Photonic Networks, 1999 Symposium on Performance Evaluation of Computer and Telecommunication Systems, Chicago IL, July 1999, pp. 84-90. 
[12] T. Stroesslin and M. Gagnaire: A Flexible MAC protocol for alloptical WDM Metropolitan Area Networks, Proceedings of IEEE IPCCC 2000, Phoenix, AZ, February 2000, pp. 113-117.

[13] R. Chipalkatti, Z. Zhang and A. S. Acampora: Protocols for Optical Star-Coupler Using WDM: Performance and Complexity Study, IEEE Journal on Selected Areas in Communications, vol. 11, no. 4, May 1993, pp. 579-589.

[14] S. Floyd and V. Jacobson: Random Early Detection Gateways for Congestion Avoidance, IEEE/ACM Transactions on Networking, vol. 1, no. 4, August 1993, pp. 397-413.

[15] David D. Clark and Wenjia Fang: Explicit Allocation of Best-Effort Packet Delivery Service, IEEE/ACM Transactions on Networking, vol. 6, no. 4, August 1998, pp. 362-373.

[16] Nada Golmie, Sandrine Masson, Gerard Pieris and David Su: Performance Evaluation of MAC Protocol Components for HFC Networks, SPIE Proceeding, vol. 2917, 1996, pp. 120-130. 